\title{
Efficient Heterogeneous Catalytic One-Pot, Three Component Synthesis of $\boldsymbol{\gamma}$-Hydroxy- $\beta$-Ketoamides
}

\author{
Hanan A. Soliman and Tamer K. Khatab* \\ Photochemistry Department, and "Organometallic and \\ Organometalloid Chemistry Department, National Research \\ Centre, Dokki, Cairo 12622, Egypt.
}

\begin{abstract}
TETRACHLOROSILANE/zinc chloride is used as an efficient heterogeneous reagent to promote a one-pot, three component synthesis of $\gamma$-Hydroxy- $\beta$-ketoamides by the reaction of dimedone, as a 1,3-diketone with various aromatic aldehydes and various nitriles, in dichloromethane at ambient temperature.
\end{abstract}

Keywords: $\gamma$-Hydroxy- $\beta$-ketoamides, Tetrachlorosilane, Heterogeneous reagent and Multicomponent reactions.

Multicomponent coupling reactions ${ }^{(1)}$ (MCRs) are attractive for parallel synthesis as large arrays of compounds with diverse substitution patterns can be prepared in one step, mostly in high yields, under mild conditions. MCRs are powerful tools in modern drug discovery and allow fast, automated and high throughput synthesis of diverse structural scaffolds required in the search of novel therapeutic and pharmacological active molecules ${ }^{(2-4)}$. Industrial and academic research have made powerful MCR strategies into one of the most efficient and cost-effective tools for combinatorial synthesis ${ }^{(5)}$. The development of novel MCRs is an intellectually challenging task, since one has to consider not only the reactivity match of the starting materials but also the reactivity of the intermediate molecules generated in situ, their compatibility and their compartmentalization ${ }^{(6)}$. Amido- or amino-ketone derivatives are significant for their biological and pharmaceutical properties ${ }^{(7)}$ and in the synthesis of antibiotic drugs such as nikkomycine or neopolyoxines ${ }^{(8)}$. The best known route for the synthesis of this class of compounds is the Dakin-West reaction ${ }^{(9 a)}$, which involves the condensation of an amino acid with acetic anhydride in the presence of a base ${ }^{(9 b)}$. Recently, other synthetic methods have been used for the formation of $\beta$-acetamidoketones through the multicomponent condensation of aryl aldehydes, enolizable ketones, and acyl chlorides in acetonitrile in the presence of Lewis or Brønsted acid catalysts such as $\mathrm{CoCl}_{2}{ }^{(10,11)}$, montmorillonite $\mathrm{K}-10$ clay ${ }^{(12)}$, silica sulfuric $\mathrm{acid}^{(13)}, \mathrm{BiCl}_{3}$ generated from $\mathrm{BiOCl}^{(14)}, \mathrm{ZrOCl}_{2} \cdot 8 \mathrm{H}_{2} \mathrm{O}^{(15)}$, heteropoly acid ${ }^{(16)}$, sulfuric acid

E.mail: tarek12_2@yahoo.com (H.A. Soliman)

Tel.: +20233371010; Fax: +20237601877.

E.mail: tamer_khatab@hotmail.com (T. K. Khatab) 
absorbed on silica gel $^{(17)}, \mathrm{Sc}(\mathrm{OTf})_{3}{ }^{(18)}$, silica supported $\mathrm{H}_{3} \mathrm{PW}_{12} \mathrm{O}_{4}{ }^{(19)}$, $\mathrm{Fe}\left(\mathrm{HSO}_{4}\right)_{3}{ }^{(20)}$, Nafion- $\mathrm{H}^{(21)}, \mathrm{NaHSO}_{4} \cdot \mathrm{H}_{2} \mathrm{O}^{(22)}$, and iron(III) chloride ${ }^{(23)}$, $\mathrm{HAPs}^{(24)}, \mathrm{ClSO}_{3} \mathrm{H}^{(25)}$, cyl chlorides ${ }^{(26)}$. Despite these valuable protocols, these methods lack the generality to produce arrays of $\beta$-amido ketones. To the best of our knowledge, there are only three known methods that have employed the MCR protocol in the reaction between demidone, aldehydes and acetonitrile (as an example from nitriles); these methods used $\mathrm{HAPs}^{(24)}, \mathrm{ClSO}_{3} \mathrm{H}^{(25)}$, or acyl chlorides $^{(26)}$ as catalysts. Therefore, introducing a new, efficient, and general method which involves the use of simple and inexpensive heterogeneous catalyst, like $\mathrm{TCS} / \mathrm{ZnCl}_{2}$ with various nitriles, aldehydes and demidone to form $\beta$-amidoketone under mild condition would be a good addition. Towards this goal, and in continuation of our investigations on the development and applications of new in situ reagents derived from tetrachlorosilane (TCS) in organic synthesis ${ }^{(27)}$, we have developed an efficient and convenient protocol for the one-pot synthesis of $\gamma$-hydroxy- $\beta$-ketoamides, biologically active druglike molecules. The reaction proceeds via a three-component one-pot condensation reaction of various aldehydes, demidone as a 1,3-diketone and nitriles including alkyl, aralkyl, aryl, and utilizing the inexpensive and readily available heterogeneous reagent $\mathrm{TCS} / \mathrm{ZnCl}_{2}$ in methylene chloride at room temperature.

\section{Results and Discussion}

The synthesis of N-[(2-hydroxy-4,4-dimethyl-6-oxo-cyclohexenyl) phenylmethyl] acetamide (4aa) in an efficient yield, was achieved through a three-component one-pot reaction of benzaldehyde $1 \mathrm{a}$, demidone 2 , and acetonitrile $3 \mathrm{a}$ by using of $\mathrm{TCS} / \mathrm{ZnCl}_{2}$ mixture in methylene chloride as a solvent at room temperature as depicted in Scheme 1.

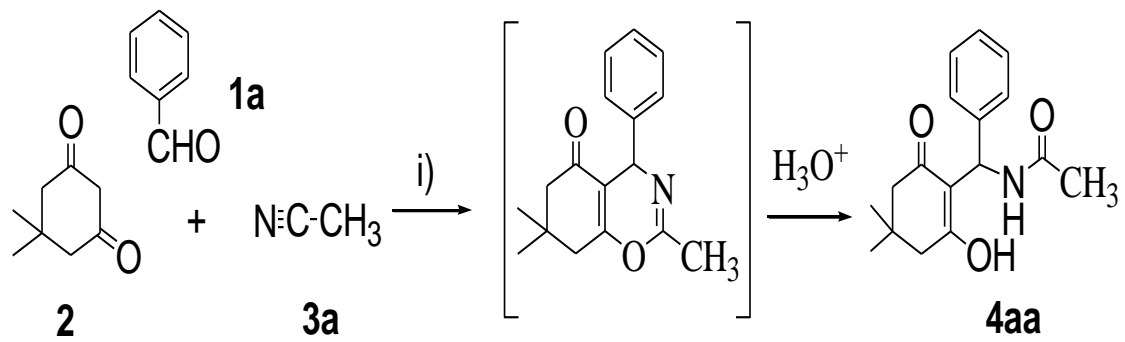

Scheme 1. Reagents and Conditions: i) $\mathrm{TCS} / \mathrm{ZnCl}_{2}, \mathrm{CH}_{2} \mathrm{Cl}_{2}$, r.t.

As a part of an ongoing study to investigate the optimum conditions for these reactions, we studied the efficacy of the catalyst type, molar ratio and solvent. The obtained results are summarized in Table 1.

Egypt. J. Chem. 57, No. 2 (2014) 
TABLE 1. Effect of catalysts and solvents on the yield and time of the one-pot synthesis of $\mathrm{N}$ [(2-hydroxy-4,4-dimethyl-6-oxo-cyclohexenyl) phenylmethyl] acetamide (4aa).

\begin{tabular}{|c|c|c|c|c|}
\hline Entry & Catalyst & Solvent & Time (hr) & $\begin{array}{c}\text { Yield } \\
(\boldsymbol{\%})\end{array}$ \\
\hline 1 & $\mathrm{TCS} / \mathrm{ZnCl}_{2}$ & $\mathrm{CH}_{2} \mathrm{Cl}_{2}$ & 2.5 & 88 \\
\hline 2 & $\mathrm{TCS} / \mathrm{ZnCl}_{2}$ & $\mathrm{CH}_{3} \mathrm{CN}$ & 5 & 60 \\
\hline 3 & $\mathrm{TCS} / \mathrm{ZnCl}{ }_{2}$ & $\mathrm{DMF} / \mathrm{CH}{ }_{3} \mathrm{CN}$ & 10 & 26 \\
\hline 4 & $\mathrm{TCS} / \mathrm{ZnCl}_{2}$ & $\mathrm{THF}$ & 10 & Nil \\
\hline 5 & $\mathrm{TCS} / \mathrm{ZnCl}_{2}$ & $\mathrm{C}_{2} \mathrm{H}_{4} \mathrm{Cl}_{2}$ & 5 & 84 \\
\hline 6 & $\mathrm{TCS}$ & $\mathrm{CH}_{2} \mathrm{Cl}_{2}$ & $10^{\Delta}$ & 76 \\
\hline 7 & $\mathrm{ZnCl}_{2}$ & $\mathrm{CH}_{2} \mathrm{Cl}_{2}$ & $10^{\Delta}$ & 45 \\
\hline 8 & $\mathrm{SnCl}_{2}$ & $\mathrm{CH}_{2} \mathrm{Cl}_{2}$ & $10^{\Delta}$ & 58 \\
\hline 9 & $\mathrm{Silica} . \mathrm{FeCl}_{3}$ & $\mathrm{CH}_{2} \mathrm{Cl}_{2}$ & $15^{\Delta}$ & 52 \\
\hline
\end{tabular}

For optimizing the reaction conditions, we examined the reaction of demidone ( 1 equiv), benzaldehyde ( 1 equiv), and acetonitrile ( 1 equiv) as a model example in the presence of $\mathrm{SiCl}_{4}-\mathrm{ZnCl}_{2}$ at room temperature in various solvents. Chlorinated solvents such as $\mathrm{CH}_{2} \mathrm{Cl}_{2}$ or $\mathrm{ClCH}_{2} \mathrm{CH}_{2} \mathrm{Cl}$ were found to be effective. The reaction was not compatible with oxygenated solvents such as diethyl ether or THF which completely inhibited the reaction, because of high oxophilicity of silicon. To determine the optimum quantity of $\mathrm{SiCl}_{4}$ and $\mathrm{ZnCl}_{2}$, the reaction was carried out in dichoromethane at room temperature using different quantities of the catalyst. The use of 2 equiv of $\mathrm{SiCl}_{4}$ and lequiv of $\mathrm{ZnCl}_{2}$ resulted in the highest yield. It is noteworthy to mention that a reaction was observed in the absence of either the Lewis acid or $\mathrm{SiCl}_{4}$ after heating. A slight excess of acetonitrile was found to be advantageous, therefore, the molar ratio of demidone, aldehyde, and nitrile was kept at 1:1:1.3, respectively, and the best results were obtained by mixing the aldehyde, demidone, nitrile, and (TCS/ $\mathrm{ZnCl}_{2}$ ) in $\mathrm{CH}_{2} \mathrm{Cl}_{2}$ as solvent. This remarkable activation in reaction rate, prompted us to explore the potential of this protocol for the synthesis of $\gamma$ hydroxy- $\beta$-ketoamides. The results are summarized in Scheme 2 and Table 2.

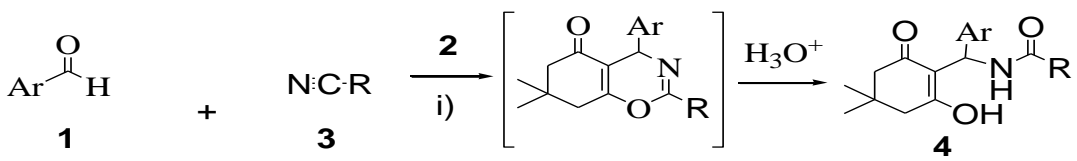

1a, Ar $=$ phenyl-

3a, $\mathbf{R}=\mathbf{M e}$ -

4aa-4ia, 4ab-4db,

$1 \mathrm{~b}, \mathrm{Ar}=4-\mathrm{Me}-\mathrm{C}_{6} \mathrm{H}_{4}-\quad 3 \mathrm{~b}, \mathrm{R}=\mathrm{Et}-$

1c, $\mathrm{Ar}=4-\mathrm{MeO}-\mathrm{C}_{6} \mathrm{H}_{4} 3 \mathrm{c}, \mathrm{R}=\mathrm{Ph}_{2} \mathrm{CH}-$

4fb, 4 bc-4ec, $4 a d$

1d, $\mathrm{Ar}=4-\mathrm{Cl}^{-\mathrm{C}_{6} \mathrm{H}_{4}-}$ 3d, $\mathrm{R}=\mathrm{Ph}-$

$1 \mathrm{e}, \mathrm{Ar}=4-\mathrm{Br}-\mathrm{C}_{6} \mathrm{H}_{4}-$

1f, $\mathrm{Ar}=2-\mathrm{Br}_{-}-\mathrm{C}_{6} \mathrm{H}_{4}-$

$1 \mathrm{~g}, \mathrm{Ar}=4-\mathrm{NO}_{2}-\mathrm{C}_{6} \mathrm{H}_{4}-$

$1 \mathrm{~h}, \mathrm{Ar}=3,4,5-(\mathrm{OMe})_{3} \mathrm{C}_{6} \mathrm{H}_{2-}$

1i, Ar $=$ naphthyl-

4bd, 4dd

Scheme 2.

Egypt. J. Chem. 57, No. 2 (2014) 
TABLE 2. Reactions of demidone 2 with a variety of aldehydes and nitriles.

\begin{tabular}{|c|c|c|c|c|c|}
\hline Entry & $\mathbf{A r}$ & $\mathbf{R}$ & Produtct & Time (hr) & Yield $^{\mathbf{a}} \mathbf{( \% )}$ \\
\hline 1 & $\mathrm{C}_{6} \mathrm{H}_{5}$ & $\mathrm{CH}_{3}$ & $4 \mathrm{aa}$ & 2.5 & 88 \\
\hline 2 & $4-\mathrm{MeC}_{6} \mathrm{H}_{5}$ & $\mathrm{CH}_{3}$ & $4 \mathrm{ba}$ & 2 & 91 \\
\hline 3 & $4-\mathrm{MeOC}_{6} \mathrm{H}_{5}$ & $\mathrm{CH}_{3}$ & $4 \mathrm{ca}$ & 1.5 & 93 \\
\hline 4 & $4-\mathrm{ClC}_{6} \mathrm{H}_{5}$ & $\mathrm{CH}_{3}$ & $4 \mathrm{da}$ & 3.5 & 85 \\
\hline 5 & $4-\mathrm{BrC}_{6} \mathrm{H}_{5}$ & $\mathrm{CH}_{3}$ & $4 \mathrm{ea}$ & 4 & 82 \\
\hline 6 & $2-\mathrm{BrC}_{6} \mathrm{H}_{5}$ & $\mathrm{CH}_{3}$ & $4 \mathrm{fa}$ & 4.5 & 81 \\
\hline 7 & $4-\mathrm{NO}_{2} \mathrm{C}_{6} \mathrm{H}_{5}$ & $\mathrm{CH}_{3}$ & $4 \mathrm{ga}$ & 5 & 75 \\
\hline 8 & $3,4,5-\left(\mathrm{MeO}_{3} \mathrm{C}_{6} \mathrm{H}_{2}\right.$ & $\mathrm{CH}_{3}$ & $4 \mathrm{ha}$ & 2 & 90 \\
\hline 9 & $\mathrm{Naphthyl}$ & $\mathrm{CH}_{3}$ & $4 \mathrm{ia}$ & 2 & 86 \\
\hline 10 & $\mathrm{C}_{6} \mathrm{H}_{5}$ & $\mathrm{C}_{2} \mathrm{H}_{5}$ & $4 \mathrm{ab}$ & 3 & 84 \\
\hline 11 & $4-\mathrm{MeC}_{6} \mathrm{H}_{5}$ & $\mathrm{C}_{2} \mathrm{H}_{5}$ & $4 \mathrm{bb}$ & 2.5 & 89 \\
\hline 12 & $4-\mathrm{MeOC}_{6} \mathrm{H}_{5}$ & $\mathrm{C}_{2} \mathrm{H}_{5}$ & $4 \mathrm{cb}$ & 2 & 90 \\
\hline 13 & $4-\mathrm{ClC}_{6} \mathrm{H}_{5}$ & $\mathrm{C}_{2} \mathrm{H}_{5}$ & $4 \mathrm{db}$ & 4 & 83 \\
\hline 14 & $2-\mathrm{BrC}_{6} \mathrm{H}_{5}$ & $\mathrm{C}_{2} \mathrm{H}_{5}$ & $4 \mathrm{fb}$ & 4.5 & 81 \\
\hline 15 & $4-\mathrm{MeC}_{6} \mathrm{H}_{5}$ & $\left(\mathrm{C}_{6} \mathrm{H}_{5}\right)_{2} \mathrm{CH}$ & $4 \mathrm{bc}$ & 4 & 92 \\
\hline 16 & $4-\mathrm{MeOC}_{6} \mathrm{H}_{5}$ & $\left(\mathrm{C}_{6} \mathrm{H}_{5}\right)_{2} \mathrm{CH}$ & $4 \mathrm{cc}$ & 3 & 95 \\
\hline 17 & $4-\mathrm{ClC}_{6} \mathrm{H}_{5}$ & $\left(\mathrm{C}_{6} \mathrm{H}_{5}\right)_{2} \mathrm{CH}$ & $4 \mathrm{dc}$ & 5 & 88 \\
\hline 18 & $4-\mathrm{BrC}_{6} \mathrm{H}_{5}$ & $\left(\mathrm{C}_{6} \mathrm{H}_{5}\right)_{2} \mathrm{CH}$ & $4 \mathrm{ec}$ & 5 & 86 \\
\hline 19 & $3,4,5-\left(\mathrm{MeO}_{3} \mathrm{C}_{6} \mathrm{H}_{2}\right.$ & $\left(\mathrm{C}_{6} \mathrm{H}_{5}\right)_{2} \mathrm{CH}$ & $4 \mathrm{hc}$ & 3 & 92 \\
\hline 20 & $\mathrm{C}_{6} \mathrm{H}_{5}$ & $\mathrm{C}_{6} \mathrm{H}_{5}$ & $4 \mathrm{ad}$ & 5 & 82 \\
\hline 21 & $4-\mathrm{MeC}_{6} \mathrm{H}_{5}$ & $\mathrm{C}_{6} \mathrm{H}_{5}$ & $4 \mathrm{bd}$ & 4 & 85 \\
\hline 22 & $4-\mathrm{ClC}_{6} \mathrm{H}_{5}$ & $\mathrm{C}_{6} \mathrm{H}_{5}$ & $4 \mathrm{dd}$ & 5.5 & 82 \\
\hline
\end{tabular}

${ }^{\mathrm{a}}$ Isolated yield

From the results obtained it was apparent that the reaction time was shorter and the yield was higher for the one-pot condensations of aldehydes or acetophenones containing an electron-donating group $\left(\mathrm{CH}_{3}, \mathrm{OCH}_{3}\right)$, relative to the unsubstituted reactants, and the presence of electron-withdrawing substituents $\left(\mathrm{Cl}, \mathrm{Br}, \mathrm{NO}_{2}\right)$ led to longer reaction times and lower yields. In addition, nitrile type also has an effect on the reaction rate; the ascending order of reaction time is acetonitrile, propyonitrile, diphenylacetonitrile and benzonitrile, respectively .

The structures of the obtained $\gamma$-hydroxy- $\beta$-ketoamides were elucidated by spectroscopic methods. The IR spectra showed peaks at $v=3397-3365,3179$ 3130 , 1657-1650, and 1634-1623 $\mathrm{cm}^{-1}$, corresponding to $\mathrm{OH}, \mathrm{NH}$, ketonic carbonyl of $\underline{\mathrm{COCH}_{2}}$, and the amidic carbonyl $\underline{\mathrm{CONH}}$ groups, respectively. The

Egypt. J. Chem. 57, No. 2 (2014) 
${ }^{1} \mathrm{H}-\mathrm{NMR}$ spectra of the synthesized products revealed singlet at $\delta=0.97-1.05$ for $2 \mathrm{CH}_{3}$, singlet and multiplet $\delta=2.14-2.68$ for $2 \mathrm{CH}_{2}$, two exchangeable protons for $\mathrm{OH}, \mathrm{NH}$, doublet signals $\delta=6.13-6.97$ for the protons $\underline{\mathrm{CHNH}}$, multiplet for aromatic protons. The ${ }^{13} \mathrm{C}$ NMR spectrum for (4ia) showed four characteristic signals at $\delta=197.6$ for the ketonic (CO), 171.2 for the enolic carbon group, at 162 for the amidic CONH carbonyl groups and 104.5 vinylic carbon; multi signals at 134.1-125.4 for aromatic carbons; 38.50 for $\mathrm{CHNH}$ and (27.1 for $2 \mathrm{CH}_{3} ; 15.5$ for quaternary carbon; $53.9,49.5$ for $2 \mathrm{CH}_{2}$ ) characteristic for demidone ring.

A plausible mechanism could be explained and demonstrated as follows, the reaction started by the addition of heterogenous catalyst $\mathrm{SiCl}_{4} / \mathrm{ZnCl}_{2}$ in a $2: 1$ molar ratio to the carbonyl group of the aldehydes, enolizable demidone as well as to cyano group in nitrile led to the formation of intermediates (I), (II), respectively, followed by [4+2] cycloaddition to afford tetrahydrobenzo[e]-1,3oxazin-5-one derivatives intermediate (III) which hydrolyzed ${ }^{(28)}$ to $\gamma$-hydroxy- $\beta$ ketoamides (4) due to the (acidic medium $\approx \mathrm{pH} 3$ ) as given in Scheme 3.

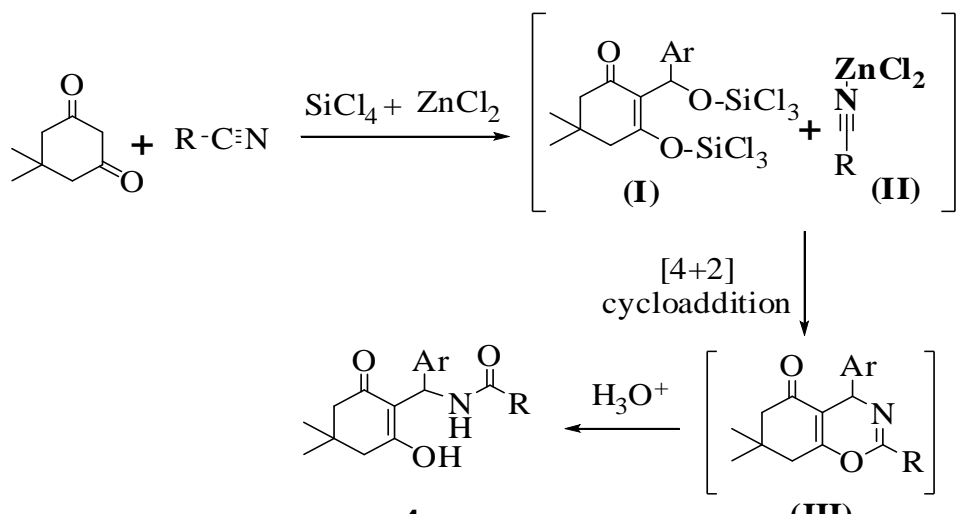

4

(III)

Scheme 3 .

Conclusion

In conclusion, a highly efficient new approach to the synthesis of $\gamma$-hydroxy$\beta$-ketoamides by the reaction between demidone, as 1,3-diketone, various aldehydes and various nitriles catalyzed by $\mathrm{TCS} / \mathrm{ZnCl}_{2}$ has been presented. The mild reaction conditions of the protocol, inexpensive reagents, ease of handling and good yield are the advantages of the present method.

\section{Experimental}

\section{Equipments}

Melting points were determined and corrected. Microanalyses were carried out by the Microanalytical Laboratory, National Research Center, Cairo, Egypt. Infrared spectra (KBr-disc) were recorded using a Jasco FT/IR-300E 
spectrometer. ${ }^{1} \mathrm{H}$ NMR and ${ }^{13} \mathrm{C}$ NMR spectra were measured in DMSO using Varian Mercury $300 \mathrm{MHz}$ with chemical shifts using TMS as standard solvent. Mass spectra were recorded on a GC/MS Finnigan SSQ 7000 spectrometer. All reactions were carried out under atmospheric conditions at room temperature.

\section{Materials}

Tetrachlorosilane (TCS), anhydrous zinc chloride were obtained from (Sigma Aldrich) company. The solvents were distilled and dried over anhydrous calcium chloride before use. Reactions were monitored by TLC on $0.25 \mathrm{~mm}$ Merck Silica gel sheets (60 GF 354) $(4 \times 2 \mathrm{~cm})$, and the spots were detected with UV light.

\section{General procedure}

To a mixture of anhydrous $\mathrm{ZnCl}_{2}(0.7 \mathrm{~g}, 5 \mathrm{mmol})$ and $\mathrm{SiCl}_{4}(1.2 \mathrm{ml}$, $10 \mathrm{mmol})$ in $\mathrm{CH}_{2} \mathrm{Cl}_{2}(20 \mathrm{ml})$ were added aldehyde $(5 \mathrm{mmol})$, dimidone $(5 \mathrm{mmol})$, and nitrile $(6.5 \mathrm{mmol})$. The reaction mixture was stirred with exclusion of moisture. The reaction monitored by (TLC) till the reaction completion, the reaction mixture was poured onto water $(100 \mathrm{ml})$, extracted with $\mathrm{CH}_{2} \mathrm{Cl}_{2}(2 \times 50 \mathrm{ml})$ and the combined organic phase dried over anhydrous $\mathrm{MgSO}_{4}$, followed by removal of the solvent under reduced pressure. The residue resulting was chromatographed on silica gel using [pet. Ether/ethyl acetate $(1: 1)]$ as eluent to give pure $\gamma$-hydroxy- $\beta$-ketoamides.

$N$-[(2-hydroxy-4,4-dimethyl -6- oxo-cyclohexenyl)- (phenyl)methyl]-acetamide (4aa)

m.p. $210{ }^{\circ} \mathrm{C}\left(\mathrm{lit}^{24}\right) R_{f} 0.36$ (pet. Ether: EtOAc 1:1), ${ }^{1} \mathrm{H}$ NMR $(300 \mathrm{MHz}$, DMSO- $d 6, \mathrm{~d} / \mathrm{ppm}$ ): 11.14 (s, $1 \mathrm{H}, \mathrm{OH}$ exchanged with $\left.\mathrm{D}_{2} \mathrm{O}\right), 8.05(\mathrm{~d}, J=9 \mathrm{~Hz}$, $1 \mathrm{H}$, NH exchanged with $\left.\mathrm{D}_{2} \mathrm{O}\right), 7.63-7.22(\mathrm{~m}, 5 \mathrm{H}, \mathrm{Ar}-\mathrm{H}), 6.83(\mathrm{~d}, J=9 \mathrm{~Hz}, 1 \mathrm{H})$ 2.68-2.60 (m, 2H), 2.23 (s, 2H), 1.99 (s, 3H), 1.05 (s, 3H), 1.01 (s, 3H).

$N$ - [(2-Hydroxy -4,4- dimethyl-6-oxo-cyclohexenyl)- (4-methylphenyl)methyl]acetamide (4ba)

m.p. $205^{\circ} \mathrm{C}\left(\mathrm{lit}^{24}\right) R_{f} 0.37$ (pet. Ether: EtOAc 1:1), ${ }^{1} \mathrm{H}$ NMR $(300 \mathrm{MHz}$, DMSO-d6, d/ppm): 11.10 (s, $1 \mathrm{H}, \mathrm{OH}$ exchanged with $\left.\mathrm{D}_{2} \mathrm{O}\right), 8.10(\mathrm{~d}, J=9 \mathrm{~Hz}$, $1 \mathrm{H}$, NH exchanged with $\left.\mathrm{D}_{2} \mathrm{O}\right), 7.63(\mathrm{~d}, J=9 \mathrm{~Hz}, 2 \mathrm{H}), 7.22(\mathrm{~d}, J=9 \mathrm{~Hz}, 2 \mathrm{H})$, $6.85(\mathrm{~d}, J=9 \mathrm{~Hz}, 1 \mathrm{H}), 2.68-2.60(\mathrm{~m}, 2 \mathrm{H}), 2.53(\mathrm{~s}, 3 \mathrm{H}), 2.23(\mathrm{~s}, 2 \mathrm{H}), 1.98(\mathrm{~s}$, $3 \mathrm{H}), 1.05(\mathrm{~s}, 3 \mathrm{H}), 1.01(\mathrm{~s}, 3 \mathrm{H})$.

\section{$N$ - [(2-Hydroxy -4,4- dimethyl- 6-oxo-cyclohexenyl) -(4-methoxyphenyl)} methyl]-acetamide (4ca)

m.p. $180{ }^{\circ} \mathrm{C}\left(\right.$ lit $\left.^{25}\right) R_{f} 0.32$ (pet. Ether: EtOAc 1:1), ${ }^{1} \mathrm{H}$ NMR $(300 \mathrm{MHz}$, DMSO-d6, d/ppm): $11.12\left(\mathrm{~s}, 1 \mathrm{H}, \mathrm{OH}\right.$ exchanged with $\left.\mathrm{D}_{2} \mathrm{O}\right), 8.10(\mathrm{~d}, J=9 \mathrm{~Hz}$, $1 \mathrm{H}$, NH exchanged with $\left.\mathrm{D}_{2} \mathrm{O}\right), 7.45(\mathrm{~d}, J=9 \mathrm{~Hz}, 2 \mathrm{H}), 7.20(\mathrm{~d}, J=9 \mathrm{~Hz}, 2 \mathrm{H})$, $6.86(\mathrm{~d}, J=9 \mathrm{~Hz}, 1 \mathrm{H}), 3.74(\mathrm{~s}, 3 \mathrm{H}), 2.68-2.69-2.63(\mathrm{~m}, 2 \mathrm{H}), 2.23(\mathrm{~s}, 2 \mathrm{H}), 2.00$ (s, 3H), $1.05(\mathrm{~s}, 3 \mathrm{H}), 1.01(\mathrm{~s}, 3 \mathrm{H})$. 
$N$ - [(4-Chlorophenyl) -(2-hydroxy -4,4-dimethyl -6-oxo- cyclohexenyl)methyl]acetamide $(4 d a)$

m.p. $208{ }^{\circ} \mathrm{C}\left(\mathrm{lit}^{24}\right){ }^{1} \mathrm{H}$ NMR (300 MHz, DMSO-d6, d/ppm): 11.10 (s, 1H, $\mathrm{OH}$ exchanged with $\left.\mathrm{D}_{2} \mathrm{O}\right), 8.08\left(\mathrm{~d}, J=9 \mathrm{~Hz}, 1 \mathrm{H}, \mathrm{NH}\right.$ exchanged with $\left.\mathrm{D}_{2} \mathrm{O}\right)$, $7.32(\mathrm{~d}, J=9 \mathrm{~Hz}, 2 \mathrm{H}), 7.20(\mathrm{~d}, J=9 \mathrm{~Hz}, 2 \mathrm{H}), 6.85(\mathrm{~d}, J=9 \mathrm{~Hz}, 1 \mathrm{H}), 2.68-2.62$ $(\mathrm{m}, 2 \mathrm{H}), 2.23$ (s, 2H), 1.98 (s, 3H), 1.05 (s, 3H), 1.01 (s, 3H).

$\mathrm{N}$-[(4-Bromophenyl)-(2-hydroxy-4,4-dimethyl-6-oxo-cyclohexenyl)methyl]-acetamide (4ea)

m.p. $203{ }^{\circ} \mathrm{C}\left(\mathrm{lit}^{25}\right){ }^{1} \mathrm{H}$ NMR (300 MHz, DMSO-d6, d/ppm): 11.13 (s, 1H, $\mathrm{OH}$ exchanged with $\left.\mathrm{D}_{2} \mathrm{O}\right), 8.11\left(\mathrm{~d}, J=9 \mathrm{~Hz}, 1 \mathrm{H}, \mathrm{NH}\right.$ exchanged with $\left.\mathrm{D}_{2} \mathrm{O}\right)$, $7.36(\mathrm{~d}, J=9 \mathrm{~Hz}, 2 \mathrm{H}), 7.22(\mathrm{~d}, J=9 \mathrm{~Hz}, 2 \mathrm{H}), 6.86(\mathrm{~d}, J=9 \mathrm{~Hz}, 1 \mathrm{H}), 2.68-2.60$ (m, 2H), 2.23 (s, 2H), 1.98 (s, 3H), 1.05 (s, 3H), 1.01 (s, 3H).

N-[(2-Bromophenyl)- (2-hydroxy -4,4- dimethyl-6-oxo- cyclohexenyl) methyl]acetamide (4fa)

m.p. $185-187{ }^{\circ} \mathrm{C}, R_{f} 0.27$ (pet. Ether: EtOAc 1:1), IR $\left(\mathrm{KBr}, \mathrm{cm}^{-1}\right) 3377$ $(\mathrm{OH}), 3175(\mathrm{NH}) 1655(\mathrm{C}=\mathrm{O}), 1632(\mathrm{C}=\mathrm{O}) .{ }^{1} \mathrm{H}$ NMR (300 MHz, DMSO-d6, d/ppm): $\delta 11.11\left(\mathrm{~s}, 1 \mathrm{H}, \mathrm{OH}\right.$ exchanged with $\left.\mathrm{D}_{2} \mathrm{O}\right), 8.10(\mathrm{~d}, J=9 \mathrm{~Hz}, 1 \mathrm{H}, \mathrm{NH}$ exchanged with $\left.\mathrm{D}_{2} \mathrm{O}\right), 7.63-7.22(\mathrm{~m}, 4 \mathrm{H}, \mathrm{ArH}), 6.85(\mathrm{~d}, J=9 \mathrm{~Hz}, 1 \mathrm{H}), 2.68-2.60$ $(\mathrm{m}, 2 \mathrm{H}), 2.23$ (s, 2H), 1.98 (s, 3H), 1.05 (s, 3H), 1.01 (s, 3H). MS (EI $70 \mathrm{eV}$ ) $m / z: 366\left(\mathrm{M}^{+}\right)$. Anal. Calcd. for $\mathrm{C}_{17} \mathrm{H}_{20} \mathrm{NO}_{3}$ (366.28): C, 55.75; H, 5.5; Br, 21.82; N, 3.82. Found: C, 55.70; H, 5.42; Br, 21.75; N, 3.75.

$\mathrm{N}$-[(2-Hydroxy-4,4-dimethyl-6-oxo-cyclohexenyl)-(4-nitrophenyl)methyl]-acetamide (4ga)

mp $202{ }^{\circ} \mathrm{C}\left(\mathrm{lit}^{26}\right){ }^{1} \mathrm{H}$ NMR (300 MHz, DMSO-d6, d/ppm): 11.15 (s, $1 \mathrm{H}, \mathrm{OH}$ exchanged with $\left.\mathrm{D}_{2} \mathrm{O}\right), 8.16\left(\mathrm{~d}, J=9 \mathrm{~Hz}, 1 \mathrm{H}, \mathrm{NH}\right.$ exchanged with $\left.\mathrm{D}_{2} \mathrm{O}\right), 7.95(\mathrm{~d}$, $J=9 \mathrm{~Hz}, 2 \mathrm{H}), 7.54(\mathrm{~d}, J=9 \mathrm{~Hz}, 2 \mathrm{H}), 6.86(\mathrm{~d}, J=9 \mathrm{~Hz}, 1 \mathrm{H}), 2.68-2.60(\mathrm{~m}, 2 \mathrm{H})$, $2.23(\mathrm{~s}, 2 \mathrm{H}), 1.98$ (s, 3H), 1.05 (s, 3H), 1.01 (s, 3H).

$N$-[(3,4,5-Trimethoxyphenyl) - (2-hydroxy -4,4- dimethyl -6-oxo-cyclohexenyl) methyl] - acetamide (4ha)

m.p. $156{ }^{\circ} \mathrm{C}, R_{f} 0.23$ (pet. Ether: EtOAc 1:1), IR $\left(\mathrm{KBr}, \mathrm{cm}^{-1}\right) 3378(\mathrm{OH})$, $3176(\mathrm{NH}) 1659(\mathrm{C}=\mathrm{O}), 1636(\mathrm{C}=\mathrm{O}) .{ }^{1} \mathrm{H}$ NMR (300 MHz, DMSO-d6, d/ppm): $\delta 11.40\left(\mathrm{~s}, 1 \mathrm{H}, \mathrm{OH}\right.$ exchanged with $\left.\mathrm{D}_{2} \mathrm{O}\right), 8.80(\mathrm{~d}, J=9.2 \mathrm{~Hz}, 1 \mathrm{H}, \mathrm{NH}$ exchanged with $\left.\mathrm{D}_{2} \mathrm{O}\right), 6.92(\mathrm{~s}, 2 \mathrm{H}, \mathrm{ArH}), 6.85(\mathrm{~d}, J=9.2 \mathrm{~Hz}, 1 \mathrm{H}), 3.76(\mathrm{~s}, 9 \mathrm{H})$, 2.68-2.60 (m, 2H), $2.23(\mathrm{~s}, 2 \mathrm{H}), 2.05(\mathrm{~s}, 3 \mathrm{H}), 1.04(\mathrm{~s}, 3 \mathrm{H}), 1.00(\mathrm{~s}, 3 \mathrm{H})$. MS (EI $70 \mathrm{eV}$ ) $\mathrm{m} / \mathrm{z}: 377\left(\mathrm{M}^{+}\right)$. Anal. Calcd. for $\mathrm{C}_{20} \mathrm{H}_{27} \mathrm{NO}_{7}$ (377.34): C, 63.64; H, 7.21; N, 3.71. Found: C, 63.60; H, 7.16; N, 3.67.

$N$-[(2-Hydroxy-4,4-dimethyl-6-oxo-cyclohexenyl)- naphthylmethyl]-acetamide (4ia)

m.p. $185^{\circ} \mathrm{C}, R_{f} 0.41$ (pet. Ether: EtOAc 1:1), IR (KBr, cm $\left.{ }^{-1}\right) 3380(\mathrm{OH})$, $3179(\mathrm{NH}) 1657(\mathrm{C}=\mathrm{O}), 1634(\mathrm{C}=\mathrm{O}) .{ }^{1} \mathrm{H}$ NMR (300 MHz, DMSO-d6, d/ppm): $\delta 11.13\left(\mathrm{~s}, 1 \mathrm{H}, \mathrm{OH}\right.$ exchanged with $\left.\mathrm{D}_{2} \mathrm{O}\right), 8.17(\mathrm{~d}, J=9 \mathrm{~Hz}, 1 \mathrm{H}, \mathrm{NH}$ exchanged 
with $\left.\mathrm{D}_{2} \mathrm{O}\right), 7.92-7.74(\mathrm{~m}, 3 \mathrm{H}), 7.49-7.36(\mathrm{~m}, 4 \mathrm{H}), 6.97(\mathrm{~d}, J=9 \mathrm{~Hz}, 1 \mathrm{H}), 2.69-$ $2.59(\mathrm{~m}, 2 \mathrm{H}), 2.24(\mathrm{~s}, 2 \mathrm{H}), 2.00(\mathrm{~s}, 3 \mathrm{H}), 1.05(\mathrm{~s}, 3 \mathrm{H}), 1.01(\mathrm{~s}, 3 \mathrm{H}) .{ }^{13} \mathrm{C}$ NMR $(75$ MHz, DMSO- $d 6$, d/ppm): 197.60, 171.20, 162.02, 134.05, 132.26, 126.52, 125.40, 104.50, 53.90, 49.50, 38.50, 27.10, 18.50, 15.50. MS (EI $70 \mathrm{eV}) \mathrm{m} / \mathrm{z}$ : $337\left(\mathrm{M}^{+}\right)$. Anal. Calcd. for $\mathrm{C}_{21} \mathrm{H}_{23} \mathrm{NO}_{3}$ (337.41): C, 74.75; H, 6.87; N, 4.15. Found: C, 74.71; H, 6.82; N, 4.10.

$N$ - [(2-Hydroxy -4,4-dimethyl-6-oxo -cyclohexenyl) -(phenyl)methyl]-propionamide (4ab)

m.p. $188-190^{\circ} \mathrm{C}, R_{f} 0.38$ (pet. ether: EtOAc 1:1), IR $\left(\mathrm{KBr}, \mathrm{cm}^{-1}\right) 3370(\mathrm{OH})$, $3120(\mathrm{NH}) 1652(\mathrm{C}=\mathrm{O}), 1625(\mathrm{C}=\mathrm{O}) .{ }^{1} \mathrm{H}$ NMR $(300 \mathrm{MHz}, \mathrm{DMSO}-d 6, \mathrm{~d} / \mathrm{ppm})$ : $\delta 11.15\left(\mathrm{~s}, 1 \mathrm{H}, \mathrm{OH}\right.$ exchanged with $\left.\mathrm{D}_{2} \mathrm{O}\right), 7.85(\mathrm{~d}, \mathrm{~J}=9 \mathrm{~Hz}, 1 \mathrm{H}, \mathrm{NH}$ exchanged with $\left.\mathrm{D}_{2} \mathrm{O}\right), 7.32-7.17(\mathrm{~m}, 5 \mathrm{H}, \mathrm{ArH}), 6.13(\mathrm{~d}, J=9 \mathrm{~Hz}, 1 \mathrm{H}), 2.38(\mathrm{~s}, 2 \mathrm{H}), 2.3-$ $2.14(\mathrm{~m}, 4 \mathrm{H}), 1.02(\mathrm{~s}, 3 \mathrm{H}), 0.97$ (s, 6H). MS (EI $70 \mathrm{eV}) \mathrm{m} / \mathrm{z}: 301\left(\mathrm{M}^{+}\right)$. Anal. Calcd. for $\mathrm{C}_{18} \mathrm{H}_{23} \mathrm{NO}_{3}$ (301.18): C, 71.73; H, 7.69; N, 4.65. Found: C, 71.70; H, $7.65 ; \mathrm{N}, 4.61$.

$N-[(2-H y d r o x y$-4,4- dimethyl -6- oxo-cyclohexenyl) -(4-methylphenyl) methyl]propionamide $(4 b b)$

m.p. $184{ }^{\circ} \mathrm{C}, R_{f} 0.37$ (pet. ether: EtOAc 1:1), IR $\left(\mathrm{KBr}, \mathrm{cm}^{-1}\right) 3377(\mathrm{OH})$, $3120(\mathrm{NH}) 1648(\mathrm{C}=\mathrm{O}), 1627(\mathrm{C}=\mathrm{O}) .{ }^{1} \mathrm{H}$ NMR (300 MHz, DMSO-d6, d/ppm): $\delta 11.19\left(\mathrm{~s}, 1 \mathrm{H}, \mathrm{OH}\right.$ exchanged with $\left.\mathrm{D}_{2} \mathrm{O}\right), 7.88(\mathrm{~d}, \mathrm{~J}=9 \mathrm{~Hz}, 1 \mathrm{H}, \mathrm{NH}$ exchanged with $\left.\mathrm{D}_{2} \mathrm{O}\right), 7.25(\mathrm{~d}, J=9 \mathrm{~Hz}, 2 \mathrm{H}), 7.14(\mathrm{~d}, J=9 \mathrm{~Hz}, 2 \mathrm{H}), 6.15(\mathrm{~d}, J=9 \mathrm{~Hz}$, $1 \mathrm{H}), 2.53(\mathrm{~s}, 3 \mathrm{H}), 2.38(\mathrm{~s}, 2 \mathrm{H}), 2.3-2.14(\mathrm{~m}, 4 \mathrm{H}), 1.02(\mathrm{~s}, 3 \mathrm{H}), 0.98(\mathrm{~s}, 6 \mathrm{H}) . \mathrm{MS}$ (EI $70 \mathrm{eV}$ ) m/z: $315\left(\mathrm{M}^{+}\right)$. Anal. Calcd. for $\mathrm{C}_{19} \mathrm{H}_{25} \mathrm{NO}_{3}$ (315.41): C, 72.35; $\mathrm{H}$, 7.99; N, 4.44. Found: C, 72.31; H, 7.94; N, 4.40.

$N$-[(2-Hydroxy-4,4-dimethyl-6-oxo- cyclohexenyl) -(4-methoxyphenyl) methyl]propionamide $(4 c b)$

m.p. $180{ }^{\circ} \mathrm{C}, R_{f} 0.27$ (pet. ether: EtOAc 1:1), IR $\left(\mathrm{KBr}, \mathrm{cm}^{-1}\right) 3376(\mathrm{OH})$, $3122(\mathrm{NH}) 1653(\mathrm{C}=\mathrm{O}), 1620(\mathrm{C}=\mathrm{O}) .{ }^{1} \mathrm{H}$ NMR (300 MHz, DMSO-d6, d/ppm): $\delta 11.11\left(\mathrm{~s}, 1 \mathrm{H}, \mathrm{OH}\right.$ exchanged with $\left.\mathrm{D}_{2} \mathrm{O}\right), 7.83(\mathrm{~d}, \mathrm{~J}=9 \mathrm{~Hz}, 1 \mathrm{H}, \mathrm{NH}$ exchanged with $\left.\mathrm{D}_{2} \mathrm{O}\right), 7.21(\mathrm{~d}, J=9.2 \mathrm{~Hz}, 2 \mathrm{H}), 7.10(\mathrm{~d}, J=9.2 \mathrm{~Hz}, 2 \mathrm{H}), 6.15(\mathrm{~d}, J=8.8$ $\mathrm{Hz}, 1 \mathrm{H}), 3.76$ (s, 3H), 2.36 (s, 2H), 2.30-2.13 (m, 4H), 1.04 (s, 3H), 0.98 (s, 6H). MS (EI $70 \mathrm{eV}) \mathrm{m} / z: 331\left(\mathrm{M}^{+}\right)$. Anal. Calcd. for $\mathrm{C}_{19} \mathrm{H}_{25} \mathrm{NO}_{4}$ (331.41): C, 68.86; H, 7.60; N, 4.23. Found: C, 68.82; H, 7.58; N, 4.16.

N-[(4-Chlorophenyl) - (2-hydroxy-4,4-dimethyl - 6-oxo - cyclohexenyl)methyl]propionamide $(4 d b)$

m.p. $192{ }^{\circ} \mathrm{C}, R_{f} 0.28$ (pet. ether: EtOAc 1:1), IR $\left(\mathrm{KBr}, \mathrm{cm}^{-1}\right) 3375(\mathrm{OH})$, $3122(\mathrm{NH}) 1650(\mathrm{C}=\mathrm{O}), 1623(\mathrm{C}=\mathrm{O}) .{ }^{1} \mathrm{H}$ NMR (300 MHz, DMSO-d6, d/ppm): $\delta 11.19\left(\mathrm{~s}, 1 \mathrm{H}, \mathrm{OH}\right.$ exchanged with $\left.\mathrm{D}_{2} \mathrm{O}\right), 7.89(\mathrm{~d}, \mathrm{~J}=9 \mathrm{~Hz}, 1 \mathrm{H}, \mathrm{NH}$ exchanged with $\left.\mathrm{D}_{2} \mathrm{O}\right), 7.30(\mathrm{~d}, J=9 \mathrm{~Hz}, 2 \mathrm{H}), 7.19(\mathrm{~d}, J=9 \mathrm{~Hz}, 2 \mathrm{H}), 6.15(\mathrm{~d}, J=9 \mathrm{~Hz}, 1 \mathrm{H})$, 2.38 (s, 2H), 2.3-2.14(m, 4H), 1.02 (s, 3H), 0.99 (s, 6H). MS (EI $70 \mathrm{eV}) \mathrm{m} / \mathrm{z}$ : 335, $337\left(\mathrm{M}^{+}, \mathrm{M}^{+}+2\right)$. Anal. Calcd. for $\mathrm{C}_{18} \mathrm{H}_{22} \mathrm{ClNO}_{3}(335.83)$ : $\mathrm{C}, 64.38 ; \mathrm{H}$, 6.60; Cl, 10.56; N, 4.17. Found: C, 64.34; H, 6.55; Cl, 10.52; N, 4.12. 
N-[(4-Bromophenyl)- (2-hydroxy-4,4- dimethyl- 6-oxo- cyclohexenyl)methyl]propionamide (4fb)

m.p. $160{ }^{\circ} \mathrm{C}, R_{f} 0.25$ (pet. ether: EtOAc 1:1), IR (KBr, cm $\left.{ }^{-1}\right) 3376(\mathrm{OH})$, $3123(\mathrm{NH}) 1649(\mathrm{C}=\mathrm{O}), 1625(\mathrm{C}=\mathrm{O}) .{ }^{1} \mathrm{H}$ NMR (300 MHz, DMSO-d6, d/ppm): $\delta 11.19\left(\mathrm{~s}, 1 \mathrm{H}, \mathrm{OH}\right.$ exchanged with $\left.\mathrm{D}_{2} \mathrm{O}\right), 7.89(\mathrm{~d}, J=9 \mathrm{~Hz}, 1 \mathrm{H}, \mathrm{NH}$ exchanged with $\left.\mathrm{D}_{2} \mathrm{O}\right), 7.62-7.23(\mathrm{~m}, 4 \mathrm{H}, \mathrm{ArH}), 6.15(\mathrm{~d}, J=9 \mathrm{~Hz}, 1 \mathrm{H}), 2.38(\mathrm{~s}, 2 \mathrm{H}), 2.3-$ $2.14(\mathrm{~m}, 4 \mathrm{H}), 1.02(\mathrm{~s}, 3 \mathrm{H}), 0.97(\mathrm{~s}, 6 \mathrm{H})$. MS (EI $70 \mathrm{eV}) \mathrm{m} / \mathrm{z}: 380,382\left(\mathrm{M}^{+}\right.$, $\mathrm{M}^{+}+2$ ). Anal. Calcd. for $\mathrm{C}_{18} \mathrm{H}_{22} \mathrm{BrNO}_{3}$ (380.28): C, 56.85; H, 5.83; Br, 21.01; N, 3.68. Found: C, 56.81; H, 5.76; Br, 20.92; N, 3.61.

$N$-[(2-Hydroxy-4,4-dimethyl-6-oxo-cyclohexenyl)-(4-methylphenyl)methyl]-2,2diphenyl acetamide $(4 b c)$

m.p. $198{ }^{\circ} \mathrm{C}, R_{f} 0.4$ (pet. ether: EtOAc 1:1), IR $\left(\mathrm{KBr}, \mathrm{cm}^{-1}\right) 3382(\mathrm{OH}), 3129$ (NH) $1658(\mathrm{C}=\mathrm{O}), 1627(\mathrm{C}=\mathrm{O}) .{ }^{1} \mathrm{H}$ NMR $(300 \mathrm{MHz}, \mathrm{DMSO}-d 6, \mathrm{~d} / \mathrm{ppm}): \delta 8.18$ (s, 1H, NH exchanged with $\left.\mathrm{D}_{2} \mathrm{O}\right), 7.52-7.10(\mathrm{~m}, 14 \mathrm{H}), 6.38(\mathrm{~d}, J=12 \mathrm{~Hz}, 1 \mathrm{H})$, $4.95\left(\mathrm{~s}, 1 \mathrm{H}, \mathrm{OH}\right.$, exchanged with $\left.\mathrm{D}_{2} \mathrm{O}\right), 3.76(\mathrm{~s}, 1 \mathrm{H}), 2.53(\mathrm{~s}, 3 \mathrm{H}), 2.20-2.26(\mathrm{~m}$, $4 \mathrm{H}), 1.00(\mathrm{~s}, 6 \mathrm{H})$. MS (EI $70 \mathrm{eV}) \mathrm{m} / \mathrm{z}: 453\left(\mathrm{M}^{+}\right)$. Anal. Calcd. for $\mathrm{C}_{30} \mathrm{H}_{31} \mathrm{NO}_{3}$ (453.57): C, 79.44; H, 6.89; N, 3.09. Found: C, 79.40; H, 6.84; N, 3.03.

$N$-[(2-Hydroxy-4,4-dimethyl -6-oxo-cyclohexenyl) - (4-methoxyphenyl) methyl]2,2-diphenyl acetamide (4cc)

m.p. $142{ }^{\circ} \mathrm{C}, R_{f} 0.35$ (pet. ether: EtOAc 1:1), $\left(\mathrm{KBr}, \mathrm{cm}^{-1}\right) 3385(\mathrm{OH}), 3130$ (NH) $1659(\mathrm{C}=\mathrm{O}), 1626(\mathrm{C}=\mathrm{O}) .{ }^{1} \mathrm{H}$ NMR $(300 \mathrm{MHz}, \mathrm{DMSO}-d 6, \mathrm{~d} / \mathrm{ppm}): \delta 8.24$ (s, 1H, NH exchanged with $\left.\mathrm{D}_{2} \mathrm{O}\right), 7.58-7.15(\mathrm{~m}, 14 \mathrm{H}), 6.37(\mathrm{~d}, J=12 \mathrm{~Hz}, 1 \mathrm{H})$, $4.97\left(\mathrm{~s}, 1 \mathrm{H}, \mathrm{OH}\right.$, exchanged with $\left.\mathrm{D}_{2} \mathrm{O}\right), 3.76(\mathrm{~s}, 1 \mathrm{H}), 3.74(\mathrm{~s}, 3 \mathrm{H}), 2.22-2.27(\mathrm{~m}$, 4H), 1.00 (s, 6H).MS (EI $70 \mathrm{eV}) \mathrm{m} / z: 469\left(\mathrm{M}^{+}\right)$. Anal. Calcd. for $\mathrm{C}_{30} \mathrm{H}_{31} \mathrm{NO}_{4}$ (469.57): C, 76.73; H, 6.65; N, 2.98. Found: C, 76.68; H, 6.60; N, 2.95.

N-[(4-Chlorophenyl)-(2-hydroxy-4,4-dimethyl-6-oxo-cyclohexenyl)methyl]-2,2diphenylacetamide $(4 d c)$

m.p. $156{ }^{\circ} \mathrm{C}, R_{f} 0.35$ (pet. ether: EtOAc 1:1), IR $\left(\mathrm{KBr}, \mathrm{cm}^{-1}\right) 3380(\mathrm{OH})$, $3130(\mathrm{NH}) 1656(\mathrm{C}=\mathrm{O}), 1628(\mathrm{C}=\mathrm{O}) .{ }^{1} \mathrm{H}$ NMR (300 MHz, DMSO-d6, d/ppm): ): $\delta 8.11\left(\mathrm{~s}, 1 \mathrm{H}, \mathrm{NH}\right.$ exchanged with $\left.\mathrm{D}_{2} \mathrm{O}\right), 7.52-7.13(\mathrm{~m}, 14 \mathrm{H}), 6.39(\mathrm{~d}, J=12$ $\mathrm{Hz}, 1 \mathrm{H}), 4.95\left(\mathrm{~s}, 1 \mathrm{H}, \mathrm{OH}\right.$, exchanged with $\left.\mathrm{D}_{2} \mathrm{O}\right), 3.76(\mathrm{~s}, 1 \mathrm{H}) 2.22-2.27(\mathrm{~m}$, 4H), $1.00(\mathrm{~s}, 6 \mathrm{H})$. MS (EI $70 \mathrm{eV}) \mathrm{m} / \mathrm{z}: 473,475\left(\mathrm{M}^{+}, \mathrm{M}^{+}+2\right)$. Anal. Calcd. for $\mathrm{C}_{29} \mathrm{H}_{28} \mathrm{ClNO}_{3}$ (473.99): C, 73.48; H, 5.95; Cl, 7.48; N, 2.96. Found: C, 73.43; $\mathrm{H}, 5.91 ; \mathrm{Cl}, 7.41 ; \mathrm{N}, 2.92$.

$\mathrm{N}$-[(4-Bromophenyl)-(2-hydroxy-4,4-dimethyl-6-oxo-cyclohexenyl)methyl]-2,2diphenyl acetamide (4ec)

m.p. $159{ }^{\circ} \mathrm{C}, R_{f} 0.33$ (pet. ether: EtOAc 1:1), IR $\left(\mathrm{KBr}, \mathrm{cm}^{-1}\right) 3377(\mathrm{OH})$, $3126(\mathrm{NH}) 1653(\mathrm{C}=\mathrm{O}), 1627(\mathrm{C}=\mathrm{O}) .{ }^{1} \mathrm{H}$ NMR (300 MHz, DMSO-d6, d/ppm): $\delta 8.10\left(\mathrm{~s}, 1 \mathrm{H}, \mathrm{NH}\right.$ exchanged with $\left.\mathrm{D}_{2} \mathrm{O}\right), 7.50-7.10(\mathrm{~m}, 14 \mathrm{H}), 6.39(\mathrm{~d}, J=12$ $\mathrm{Hz}, 1 \mathrm{H}), 4.95\left(\mathrm{~s}, 1 \mathrm{H}, \mathrm{OH}\right.$, exchanged with $\left.\mathrm{D}_{2} \mathrm{O}\right), 3.76(\mathrm{~s}, 1 \mathrm{H}) 2.22-2.28(\mathrm{~m}$, 4H), $1.00(\mathrm{~s}, 6 \mathrm{H})$. MS (EI $70 \mathrm{eV}) \mathrm{m} / \mathrm{z}: 518,520\left(\mathrm{M}^{+}, \mathrm{M}^{+}+2\right)$. Anal. Calcd. for 
$\mathrm{C}_{29} \mathrm{H}_{28} \mathrm{BrNO}_{3}$ (518.44): C, 67.18; H, 5.44; Br, 15.41; N, 2.7. Found: C, 67.13; $\mathrm{H}, 5.40 ; \mathrm{Br}, 15.36 ; \mathrm{N}, 2.66$.

$N$-[(3,4,5-Trimethoxyphenyl)- (2-hydroxy -4,4- dimethyl - 6-oxo- cyclohexenyl) methyl] -2,2-diphenylacetamide (4hc)

m.p. $160{ }^{\circ} \mathrm{C}, R_{f} 0.26$ (pet. ether: EtOAc 1:1), IR (KBr, cm $\left.{ }^{-1}\right) 3382(\mathrm{OH}), 3132$ (NH) $1653(\mathrm{C}=\mathrm{O}), 1626(\mathrm{C}=\mathrm{O}) .{ }^{1} \mathrm{H}$ NMR (300 MHz, DMSO- $\left.d 6, \mathrm{~d} / \mathrm{ppm}\right): \delta 8.16$ (s, $1 \mathrm{H}, \mathrm{NH}$ exchanged with $\left.\mathrm{D}_{2} \mathrm{O}\right), 7.52-7.07(\mathrm{~m}, 12 \mathrm{H}), 6.35(\mathrm{~d}, J=12 \mathrm{~Hz}, 1 \mathrm{H})$, $4.93\left(\mathrm{~s}, 1 \mathrm{H}, \mathrm{OH}\right.$, exchanged with $\left.\mathrm{D}_{2} \mathrm{O}\right), 3.77(\mathrm{~s}, 9 \mathrm{H}), 3.75(\mathrm{~s}, 1 \mathrm{H}), 2.24-2.28(\mathrm{~m}$, $4 \mathrm{H}), 1.00(\mathrm{~s}, 6 \mathrm{H})$. MS (EI $70 \mathrm{eV}) \mathrm{m} / z: 529\left(\mathrm{M}^{+}\right)$. Anal. Calcd. for $\mathrm{C}_{32} \mathrm{H}_{35} \mathrm{NO}_{6}$ (529.62): C, 72.57; H, 6.66; N, 2.64. Found: C, 72.53; H, 6.61; N, 2.61.

$N$-[(2-Hydroxy -4,4-dimethyl-6-oxo-cyclohexenyl) - (phenyl)methyl]-benzamide (4ad)

m.p. $170{ }^{\circ} \mathrm{C}, R_{f} 0.29$ (pet. ether: EtOAc 1:1), IR (KBr, cm $\left.{ }^{-1}\right) 3368(\mathrm{OH})$, $3135(\mathrm{NH}) 1656(\mathrm{C}=\mathrm{O}), 1633(\mathrm{C}=\mathrm{O}) .{ }^{1} \mathrm{H}$ NMR (300 MHz, DMSO-d6, d/ppm): $\delta 10.06\left(\mathrm{~s}, 1 \mathrm{H}, \mathrm{OH}\right.$ exchanged with $\left.\mathrm{D}_{2} \mathrm{O}\right), 8.02(\mathrm{~d}, J=8.2 \mathrm{~Hz}, 1 \mathrm{H}, \mathrm{NH}$ exchanged with $\left.\mathrm{D}_{2} \mathrm{O}\right), 7.96-7.05(\mathrm{~m}, 9 \mathrm{H}), 5.55(\mathrm{~d}, J=8 \mathrm{~Hz}, 1 \mathrm{H}), 2.00-2.04(\mathrm{~m}$, 4H) $1.10(\mathrm{~s}, 6 \mathrm{H})$. MS (EI $70 \mathrm{eV}) \mathrm{m} / z: 349\left(\mathrm{M}^{+}\right)$. Anal. Calcd. for $\mathrm{C}_{22} \mathrm{H}_{23} \mathrm{NO}_{3}$ (349.42): C, 75.62; H, 6.63; N, 4.01. Found: C, 75.56; H, 6.58; N, 3.97.

$N$-[(2-Hydroxy -4,4-dimethyl-6-oxo-cyclohexenyl)- (4-methylphenyl) methyl]benzamide $(4 b d)$

m.p. $185^{\circ} \mathrm{C}, R_{f} 0.3$ (pet. ether: EtOAc 1:1), IR (KBr, $\left.\mathrm{cm}^{-1}\right) 3367(\mathrm{OH}), 3133$ (NH) $1654(\mathrm{C}=\mathrm{O}), 1632(\mathrm{C}=\mathrm{O}) .{ }^{1} \mathrm{H}$ NMR (300 MHz, DMSO-d6, d/ppm): $\delta$ $10.06\left(\mathrm{~s}, 1 \mathrm{H}, \mathrm{OH}\right.$ exchanged with $\left.\mathrm{D}_{2} \mathrm{O}\right), 8.02(\mathrm{~d}, J=8 \mathrm{~Hz}, 1 \mathrm{H}, \mathrm{NH}$ exchanged with $\left.\mathrm{D}_{2} \mathrm{O}\right), 7.96-7.05(\mathrm{~m}, 9 \mathrm{H}), 5.56(\mathrm{~d}, J=8 \mathrm{~Hz}, 1 \mathrm{H}), 2.53(\mathrm{~s}, 3 \mathrm{H}), 1.98-2.03$ $(\mathrm{m}, 4 \mathrm{H}) 1.10(\mathrm{~s}, 6 \mathrm{H}) .{ }^{13} \mathrm{C}$ NMR (75 MHz, DMSO-d6): $\delta$ 197.60, 170.20, 162.00, 139.05, 133.26, 127.22, 129.00, 104.50, 53.60, 49.30, 40.50, 27.10, 15.50. MS (EI $70 \mathrm{eV}$ ) $\mathrm{m} / \mathrm{z}: 363\left(\mathrm{M}^{+}\right)$. Anal. Calcd. for $\mathrm{C}_{23} \mathrm{H}_{25} \mathrm{NO}_{3}$ (363.45): C, 76.01; $\mathrm{H}$, 6.93 ; N, 3.85. Found: C, 75.95; H, 6.90; N, 3.81.

$N$-[(4-Chlorophenyl)- (2-hydroxy-4,4-dimethyl-6-oxo-cyclohexenyl) methyl]benzamide $(4 d d)$

m.p. $190{ }^{\circ} \mathrm{C}, R_{f} 0.27$ (pet. ether: EtOAc 1:1), IR (KBr, cm $\left.{ }^{-1}\right) 3369(\mathrm{OH})$, $3136(\mathrm{NH}) 1659(\mathrm{C}=\mathrm{O}), 1636(\mathrm{C}=\mathrm{O}) .{ }^{1} \mathrm{H}$ NMR (300 MHz, DMSO-d6, d/ppm): $\delta 10.11\left(\mathrm{~s}, 1 \mathrm{H}, \mathrm{OH}\right.$ exchanged with $\left.\mathrm{D}_{2} \mathrm{O}\right), 8.09(\mathrm{~d}, J=8 \mathrm{~Hz}, 1 \mathrm{H}, \mathrm{NH}$ exchanged with $\left.\mathrm{D}_{2} \mathrm{O}\right), 7.98-7.00(\mathrm{~m}, 9 \mathrm{H}), 5.58(\mathrm{~d}, J=8 \mathrm{~Hz}, 1 \mathrm{H}), 2.00-2.05(\mathrm{~m}, 4 \mathrm{H}) 1.10(\mathrm{~s}$, $6 \mathrm{H})$. MS (EI $70 \mathrm{eV}) \mathrm{m} / \mathrm{z}: 383,385\left(\mathrm{M}^{+}, \mathrm{M}^{+}+2\right)$. Anal. Calcd. for $\mathrm{C}_{22} \mathrm{H}_{22} \mathrm{ClNO}_{3}$ (383.87): C, 68.83; H, 5.78; Cl, 9.24; N, 3.65. Found: C, 68.75; H, 5.73; Cl, $9.18 ; \mathrm{N}, 3.60$.

\section{References}

1. (a) Domling, A. and Ugi, I., Multicomponent reactions with isocyanides. Angew. Chem. Int. Ed., 39, 3168 (2000); (b) Thomson, L.A. and Ellman, J.A., Synthesis and applications of small molecule libraries. Chem. Rev., 96, 555 (1996); (c) Nair,

Egypt. J. Chem. 57, No. 2 (2014) 
V., Rajesh, C., Vinod, A. U., Bindu, S., Sreekanth, A. R., Mathen, J. S. and Balagopala, L., Strategies for heterocyclic construction via novel multicomponent reactions based on isocyanides and nucleophilic carbenes. Acc. Chem. Res. 36, 899 (2003).

2. (a) Weber, L., Multi-component reactions and evolutionary chemistry. Drug Discovery Today, 7, 143 (2002); (b) Hulme, C. and Gore, V., Multi-component reactions : Emerging chemistry in drug discovery from xylocain to crixivan. Curr. Med. Chem., 10, 51 (2003); (c) Tempest, P. A., The chemistry of heterocycles lies at the heart of drug discovery. Curr. Opin. Drug Discovery Dev. 8, 776 (2005).

3. Dondoni, A. and Massi, A., Design and synthesis of new classes of heterocyclic Cglycoconjugates and carbon-linked sugar and heterocyclic amino acids by asymmetric multicomponent reactions (AMCRs). Acc. Chem. Res. 39, 451 (2006).

4. (a) Xue, D., Chen, Y.-C., Wang, Q.-W., Cun, L.-F., Zhu, J. and Deng, J.-G., Asymmetric direct vinylogous Michael reaction of activated alkenes to nitroolefins catalyzed by modified cinchona alkaloids. Org. Lett., 7, 5293 (2005); (b) Xue, D., Li, J., Zhang, Z.-T. and Deng, J.-G., Efficient method for the synthesis of polysubstituted benzenes by one-pot tandem reaction of vinyl malononitriles with nitroolefins. J. Org. Chem., 72, 5443 (2007); (c) Pratap, R. and Ram, V. J., An efficient de novo synthesis of partially reduced phenanthrenes through C-C insertion. J. Org. Chem., 72, 7402 (2007).

5. (a) Zhu, J. and Bienaymé, H., (Ed.), In: Multicomponent Reactions, Wiley-VCH: Weinheim, Germany, (2005); (b) Domling, A., Isocyanide based multi component reactions in combinatorial chemistry. Comb. Chem. High Throughput Screen. 1, 1, (1998) (c) Bienayme, H., Hulme, C., Oddon, G. and Schmitt, P., Maximizing synthetic Efficiency: multi-component transformations lead the way. Chem. Eur. J. 6, 3321 (2000).

6. Ramon, D. J. and Yus, M., Asymmetric multicomponent reactions (AMCRs): the new frontier. Angew. Chem., Int. Ed. 44, 1602 (2005).

7. (a) Casimir, J. R., Turetta, C., Ettouati, L. and Paris, J., First application of the Dakin-West reaction to fmoc chemistry: Synthesis of the ketomethylene tripeptide fmoc-N $\alpha-\mathrm{Asp}(\mathrm{tBu})-\left(\mathrm{R}, \mathrm{S}-\mathrm{Tyr}(\mathrm{tBu}) \Psi\left(\mathrm{CO}-\mathrm{CH}_{2}\right) \mathrm{Gly}-\mathrm{OH}\right.$. Tetrahedron Lett., 36, 4797 (1995); (b) Godfrey, A. G.,Brooks, D. A., Hay, L. A., Peters, M., McCarthy, J. R. and Mitchell, D., Mitchell, application of the Dakin-West reaction for the synthesis of oxazole-containing dual PPARalpha/gamma agonists. J. Org. Chem. 68, 2623 (2003).

8. (a) Dahn, U., Hagenmaier, H., Ho“hne, H., König, W. A., Wolf, G. and Za"hner, H., Metabolic products of microorganisms synthesis and biosynthesis of substituted tryptanthrins. Arch. Microbiol. 107, 249 (1976); (b) Kobinata, K., Uramoto, M., Nishii, M., Kusakabe, H., Nakamura, G. and Isono, K., New chitin synthetase inhibitors. Agric. Biol. Chem. 44, 1709 (1980).

9. (a) Dakin H. D. and West, R., The reaction of -amino acids with acetic anhydride to give -acetamido ketones via azalactone intermediates. J. Biol. Chem. 78, 745 (1928); (b) Buchanan, G. L., the Dakin-West reaction. Chem. Soc. Rev. 17, 91 (1988).

Egypt. J. Chem. 57, No. 2 (2014) 
10. Mukhopadhyay, M., Bhatia, B. and Iqbal, J., Cobalt catalyzed multiple component condensation route to $\beta$-acetamido carbonyl compound libraries. Tetrahedron Lett. 381083 (1997).

11. Bhatia, B., Reddy, M. M. and Iqbal, J., Highly stereoselective synthesis of $\alpha, \beta$ unsaturated ketones by $\mathrm{CeCl}_{3}$ mediated addition of grignard reagents to $\beta$-enamino ketones. J. Chem. Soc. Chem. Commun. 713 (1994).

12. Bahulayan, D., Das, S. K. and Iqbal, J., Montmorillonite K10 clay: An efficient catalyst for the one-pot stereoselective synthesis of beta-acetamido ketones. J. Org. Chem. 685735 (2003).

13. Khodaei, M. M., Khosropour, A. R. and Fattahpour, P., A modified procedure for the Dakin-West reaction: an efficient and convenient method for a one-pot synthesis of $\beta$-acetamido ketones using silica sulfuric acid as catalyst. Tetrahedron Lett. 46, 2105 (2005).

14. Ghosh, R., Maity, S. and Chakraborty, A., One-pot multicomponent synthesis of $\beta$-acetamido ketones based on $\mathrm{BiCl}_{3}$ generated in situ from the procatalyst $\mathrm{BiOCl}$ and acetyl chloride, Synlett, 115 (2005).

15. Ghosh, R., Maity, S., Chakraborty, A., Chakraborty, S. and Mukherjee, A. K. $\mathrm{ZrOCl}_{2} \cdot 8 \mathrm{H}_{2} \mathrm{O}$ : An efficient Lewis acid catalyst for the one-pot multicomponent synthesis of $\beta$-acetamido ketones. Tetrahedron, 62, 4059 (2006).

16. Rafiee, E., Tork, F. and Joshaghani, M., Heteropoly acids as solid green Brønsted acids for a one-pot synthesis of beta-acetamido ketones by Dakin-West reaction. Bioorg. Med. Chem. Lett. 16, 1221 (2006).

17. Yakaiah, T., Reddy, G., Lingaiah, B. P. V., Narsaiah, B. and Rao, P. S., Silica sulphuric acid: An efficient and recyclable catalyst for the synthesis $\beta$ - acetamido ketones in single pot. Synth. Commun., 35, 1307 (2005).

18. Pandey, G., Singh, R. P., Garg, A. and Singh, V. K., Synthesis of Mannich type products via a three-component coupling reaction. Tetrahedron Lett. 46, 2137 (2005).

19. Rafiee, E., Shahbazi, F., Joshaghani, M. and Tork, F., The silica supported $\mathrm{H}_{3} \mathrm{PW}_{12} \mathrm{O}_{40}$ (a heteropoly acid) as an efficient and reusable catalyst for a one-pot synthesis of $\beta$-acetamido ketones by Dakin-West reaction. J. Mol. Catal. A: Chem. 242, 129 (2005).

20. Shaterian, H. R., Yarahamadi, H. and Ghashang, M., One-pot preparation of $\beta$ amido ketones and esters infour-component condensation reaction using ferric hydrogensulfate as effective and reusable catalyst. Arkivoc, (xvi), 298 (2007).

21. Yakaiah, T., Lingaiah, B. V. P., Venkat Reddy, G., Narsaiah, B. and Shanthan Rao, P., Perfluorinated resin-sulfonic acid (Nafion-H): an efficient, environment friendly and recyclable heterogeneous catalyst for the one-pot multicomponent synthesis of $\beta$-acetamido ketones. Arkivoc, (xiii), 227 (2007). 
22. Hassankhani, A., Maghsoodlou, M. T. and Habibi-Khorassani, S. M., $\mathrm{NaHSO}_{4} \cdot \mathrm{H}_{2} \mathrm{O}$ as an efficient and eco-friendly catalyst for the one-pot multicomponent synthesis of $\beta$-acetamido ketones under mild and heterogeneous conditions. Arkivoc, (ii), 134 (2008).

23. Khan, A. T., Parvin, T. and Choudhury L. H., Iron(III) chloride-catalyzed convenient one-pot synthesis of $\beta$-acetamido carbonyl compounds. Tetrahedron, $\mathbf{6 3}$ 5593 (2007).

24. Javid, J., Heravi, M. M. and Bamoharram, F. F., One-pot three-component synthesis of $\beta$-acetamido carbonyl compounds catalyzed by heteropoly acids. Monatsh. Chem. 143, 831 (2012).

25. Abbasinejad M. A., Hassanabadi, A. and Charkhati, K., Three-component reaction of 5,5-dimethylcyclohexan-1,3-dione, aromatic aldehydes and acetonitrile in the presence of chlorosulfonic acid forming $\mathrm{N}$-[(2-hydroxy-4,4-dimethyl-6oxocyclohexenyl)-aryl-methyl]-acetamide. Synth. Commun. 39, 4289 (2009).

26. Oskooie, H. A., Heravi, M. M., Tahershamsi, L., Sadjadi, S. and Tajbakhsh, M., synthesis of new $\beta$-acetamido carbonyl derivatives using cellulose sulfuric acid as an efficient catalysts. Synth. Commun. 40, 1772 (2010).

27. (a) El-Bayouki, K. A. M., Khatab, T. K., Basyouni, W. M., El-Basyoni,F. A., Hamed, A. R. and Mostafa, E. A., Efficient and expeditious synthesis of pyranopyrimidines, multi-substituted $\gamma$-pyrans, and their antioxidant activity. J. Het. Chem. 51, 106 (2014). (b) Soliman, H. A. and Salama, T. A., Silicon-mediated highly efficient synthesis of 1,8-dioxo-octahydroxanthenes and their transformation to novel functionalized pyrano-tetrazolo[1,5-a]azepine derivatives, Chin. Chem. Lett. 24(x) 404 (2014). (c) Khatab, T. K., El-Bayouki, K. A. M. and Basyouni, W. M., An efficient synthesis of $\beta$-acylureas via a three-component, one-pot synthesis using $\mathrm{TCS} / \mathrm{ZnCl}_{2}$. Tetrahedron Lett. 52, 1448 (2011).

28. Lukas, W., Jason, H., Martina, W. and Tomas, H., Synthesis of 1,2- and 1,4amino alcohols from 1,3-dienes via oxazines rearrangements of 1,4-amino alcohol derivatives to oxazolines. Tetrahedron, 66, 3761 (2010).

(Received 24/3/2014; accepted 29/6/2014) 


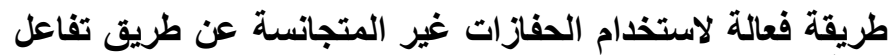

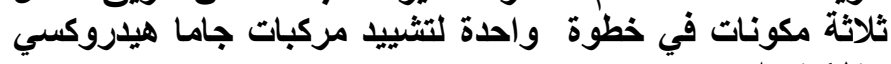

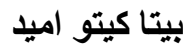

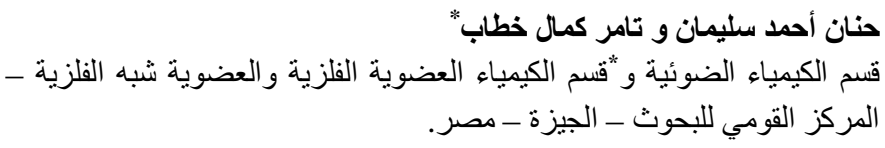

استخدام رابع كلوريد السيليكون وثاني كلوريد الزنلك كحفاز غير متجانس فعال

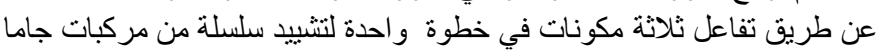

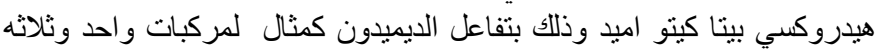

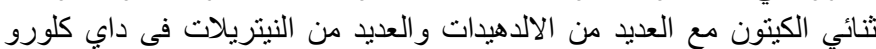
ميثان كمذيب عند درجة حرارة الغين من الإنة 